\title{
The dynamic mechanical properties of cellularised aggregates
}

\author{
Khalilgharibi $\mathrm{N}^{1,2^{*}}$,Fouchard $\mathrm{J}^{1^{*}}$, Recho $\mathrm{P}^{3^{*}}$, Charras $\mathrm{G}^{1,4,5+}$, Kabla $\mathrm{A}^{3+}$ \\ ${ }^{1}$ London Centre for Nanotechnology, University College London \\ ${ }^{2}$ CoMPLEX PhD program, University College London, UK \\ ${ }^{3}$ Department of Mechanical Engineering, Cambridge University, UK \\ ${ }^{4}$ Department of Cell and Developmental Biology, University College London, UK \\ ${ }^{5}$ Institute for the Physics of Living Systems, University College London, UK \\ * These authors contributed equally \\ +correspondence to Alexandre Kabla (ajk61@cam.ac.uk) or Guillaume Charras (g.charras@ucl.ac.uk).
}

\begin{abstract}
Cellularised materials are composed of cells interfaced through specialised intercellular junctions that link the cytoskeleton of one cell to that of its neighbours allowing for transmission of forces. Cellularised materials are common in early development and adult tissues where they can be found in the form of cell sheets, cysts, or amorphous aggregates and in pathophysiological conditions such as cancerous tumours. Given the growing realisation that forces can regulate cell physiology and developmental processes, understanding how cellularised materials deform under mechanical stress or dissipate stress appear as key biological questions. In this review, we will discuss the dynamic mechanical properties of cellularised materials devoid of extracellular matrix.
\end{abstract}




\section{Introduction}

Cellularised materials are composed of cells interfaced through specialised intercellular junctions. These link the cytoskeleton of one cell to that of its neighbours allowing for transmission of forces over large length-scales. Cellularised materials are common in early development and adult tissues where they can be found in the form of cell sheets, cysts, or amorphous aggregates and in pathophysiological conditions such as cancerous tumours. In this review, we will discuss the mechanical properties of cellularised materials devoid of extracellular matrix (ECM). Early in development, the ECM is either absent or present in such scant quantities that it may play a polarisation role rather than a mechanical one [1-4]. In contrast, in adult tissues, the ECM tends to dominate mechanical responses and its remodelling by cells can conceptually be thought of as a very slow process.

The mechanics of cellularised materials are influenced by organisation and dynamics at both the molecular- and the cellular-scale. The relative importance of these two length-scales remains an open question and likely depends on what time-scale is being considered. For example, at time-scales shorter than a second, the contributions of genetic and biological regulatory pathways can safely be ignored whereas, for longer time-scales, biochemistry and signalling must to be taken into account.

In this review, we will first summarise experiments examining the rheology of cellularised materials, then we will turn to the respective roles of molecular- and cellular-scale phenomena in tissue mechanics, and finally, we will discuss the various theoretical approaches used to investigate tissue mechanics.

\section{The rheology of cellularised materials}

Cellularised aggregates possess complex mechanical properties. Indeed, dependent on the duration over which stress is applied, they behave either as liquids or solids. On time-scales of the order of 10s, cellular aggregates behave elastically, recovering their original shape after a transient application of force [5-8]. Similar relaxation times have also been measured in epithelia in vivo by monitoring the shape relaxation of a large tissue domain after its separation from the Drosophila pupa dorsal thorax epithelium by laser ablation [9]. Such consistency in mechanical relaxation time-scales may reflect the large degree of conservation of proteic constituents and molecular-scale organisation of intercellular junctions across species. An interesting feature of cellularised aggregates is that they often display non-linear behaviours. For example, monolayers devoid of a substrate display two clear regimes with different stiffnesses when subjected to increasing extension [10], suggesting that some mechanical elements may only be solicited above a certain threshold. In contrast with this short time-scale elastic response, on long time-scales (tens of minutes to hours), cell aggregates exhibit a liquid-like behavior [5,8]. This tissue fluidity, often associated with cellular-scale rearrangements, plays a central role during morphogenetic movement in embryogenesis [11]. At intermediate time-scales (typically tens of minutes), living materials, alongside most soft materials such as polymer melts, gels or elastomers, are neither purely elastic nor purely liquid. Indeed, experimental work by many groups has shown that the stress imposed on the cellularised material cannot be entirely dissipated implying that some cellular components must bear part of the tension applied $[10,12,13]$. Another manifestation of this dual nature is the slow flow (or creep) observed in some cellularised tissues when subjected 
to constant tension over minute time-scales [10]. Despite physiological relevance for the respiratory and cardiovascular systems, epithelial tissue rheology on sub-second time-scales has so far been insufficiently probed.

To describe the dynamic mechanical behaviours of tissues, linear rheology is often adopted as a conceptual framework (for an introduction see $[14,15]$ ). This relies on describing a material's dynamic mechanical properties based on arrangements of standard mechanical components: springs that are elastic, dashpots that are viscous, and more complex active elements. Springs are parametrized by a stiffness that sets the force necessary to extend them and, when the force applied is removed, they return to their initial shape (Fig 1A). Dashpots are parametrized by a viscosity that sets the rate at which they can be deformed; in contrast to springs, they do not recover their initial length if the applied force returns to zero. Combinations of springs and dashpots arranged in series or in parallel can be used to mimic the dynamic mechanical properties of just about any passive material in a linear regime. For instance, a spring in parallel with a dashpot behaves on short time-scales like a fluid, but on long time-scales like a solid, with a transition between the two regimes controlled by the ratio between the viscosity and the stiffness (Fig 1A). A faithful description of the dynamic mechanical properties of cellularised materials often necessitates the inclusion of multiple rheological elements to take into account aspects of the macroscale behavior that emanate from the molecular- and cellular-scales at different time-scales and different strain regimes (Fig 1B). The larger the number of components, the richer and more complex the behaviour can be. An extreme example is power law behaviour, which has been observed in single cells as well as cellularised materials $[10,16,17]$. It implies either the existence of a large number of relaxation timescales due to the multitude of distinct biochemical and physical phenomena taking place within the material or a hierarchical spatial organization of the same units within the tissue (Fig 1B). Another fundamental challenge comes from the fact that constant metabolic energy generation allows for the generation of active stresses and strains inside the material due for instance to cell growth or contractility which must to be added to the passive rheological description. Though it is usually possible to design a rheological model that accurately fits the observed behavior of tissues, the challenge resides in identifying the molecular- or cellular-scale origin of the observed mechanical response.

\section{Mechanical behaviours of tissues arising directly from cellular mechanics}

As cellularised materials are constituted of cells, cellular rheological properties naturally influence those of the aggregate. We now discuss what is currently known about the links between rheological properties of single cells and those of cellularised aggregates.

The actomyosin cytoskeleton is widely thought to be the most important determinant of cellular mechanical properties while microtubules are mostly involved in intracellular transport and cell division. Consequently, actomyosin rheology has been the subject of intense study (for a review $[18,19]$ ). Filaments within the actin cytoskeleton continuously turnover on time-scales of minutes. They are connected to one another through specialized crosslinkers whose attachment-detachment endows crosslinked actin networks with viscous liquid behaviours, dictates their relaxation time, and allows them to rearrange to adapt to new configurations [20-24]. Myosin motors play a dual role as crosslinkers and force dipoles generating tension within filaments. At time-scales long compared to F-actin turnover (>1min), crosslinked actomyosin networks generate a constant tension. Overall, the exact 
rheological behaviour of cellular actin networks depends on their turnover, connectivity and contractility, all of which can be modulated by signaling [25]. In a continuum limit and close to thermodynamic equilibrium, the cytoskeleton is well understood within the theoretical framework of active gels (reviewed in [26]). Many studies on monolayers in embryos and in vitro suggest that as in isolated cells, myosin contractility plays a central role in tissue mechanics and dynamics $[9,10,27]$. In particular, the actin cortex, a submembranous layer of actin, myosin, and actin-binding proteins present in most isolated animal cells [28] has been shown to reorganise embryonic epithelia via pulses of contractility arising in the medial apical cortex of individual cells $[29,30]$. To add further complexity, the mechanical properties of cells can be altered by forces through mechanotransduction [31].

Though much work has concentrated on F-actin, there is mounting evidence that intermediate filaments play an important role in the mechanics of isolated cells [32-35]. Intermediate filaments are strain-stiffening [36] and can withstand far higher strain than Factin [37] (reviewed in [37]). In isolated cells, keratin or vimentin depletion both lead to a decrease in cytoplasmic elasticity [32,33]. Interestingly, the turnover of intermediate filaments occurs on time-scales on the order of hours [38]. Thus intermediate filaments appear to act on strain magnitudes and time-scales clearly separated from those of actomyosin which may indicate that they contribute to mechanics under different circumstances.

At the cellular level, microtubules do not possess a large enough tensile stiffness to significantly contribute to cell rheology despite a persistence length orders of magnitude larger than F-actin or intermediate filaments [37]. However, microtubules are resistant to compression of physiological order of magnitude when crosslinked and braced by the surrounding actomyosin network [39] and they may thus mechanically stabilize the cytoskeleton. However, most current experimental evidence points to microtubules playing only an indirect role in cell mechanics by sequestering Rho-GEFs at their growing ends [40].

At very short time-scales (shorter than a few seconds), complex rheological behaviours arise from the interaction between the solid and liquid phase of the cell, crowding, and the plethora of biochemical reactions taking place within the cell (reviewed in [41]). Conceptually, cells can be thought of as a porous solid cytoskeleton immersed in liquid cytosol. When force is abruptly applied to a cell $(<1 \mathrm{~s})$, the solid and liquid phases both bear some of the load. Movement of the solid phase towards its mechanical equilibrium position necessitates redistribution of interstitial water which results in stress dissipation [42]. Though poroelasticity and crowding likely play a role in tissue rheology, their respective roles have yet to be ascertained.

\section{Mechanics of cellularised aggregates arising from multi-cellular structures}

Though some of the mechanical properties of cellular aggregates directly stem from single cells, others arise due to the integration of cells into a tissue. At short time-scales and small deformations, the elastic moduli of cell aggregates range from a value similar to that of single cells to a few orders of magnitude higher for the same type of mechanical test $[10,13,43]$. This higher range of moduli may point to an important role for cell-cell junctions in stiffening the material, either through a more optimal organisation for load bearing and stress transmission or signaling downstream of intercellular junction assembly. As a consequence, 
the role of intercellular junctions in the mechanics of cellularised tissues has attracted considerable interest.

The best understood junctions are adherens junctions that link the actin cytoskeleton of adjacent cells via cadherin-catenin complexes (reviewed in $[44,45]$ ). In combination with cortical tension, adherens junctions integrate the mechanical tension generated in single cells through junctional myosin contractility to yield an overall tissue tension $[9,10]$. Junctions act as hubs for signaling and polarity, making them prime locations for mechanosensation and mechanotransduction. Interestingly, the time-scale over which mechanotransduction takes place in junctions [46] and the cytoskeleton [47] is similar to the time-scale over which cellularised aggregates relax following stress application. Thus, mechanotransduction may represent a selected adaptation process that allows tissues to sustain stresses at intermediate time-scales until cellular-scale mechanical adaptation processes can occur. The creep observed on time-scales of a few minutes in cellularised tissues may have its origin in biochemical turnover within intercellular junctions in the form of viscous flow of adherens junctions within the plane of the membrane or a fast attachment/detachment of cadherincatenin complexes from the junctional cytoskeleton that would generate shear viscosity. At longer time-scales, junctional remodeling offers a mechanism through which some of the cellular-scale adaptation processes can take place to allow remodeling of tissue organisation through cell rearrangements, cell division, and cell extrusion $[48,49]$. An important current question in the field is to understand the relative importance of junctional actomyosin, apical cortical tension due to medial actomyosin, and cadherin-mediated intercellular adhesion in setting tissue mechanics.

Desmosomes link the keratin intermediate filaments (IFs) of neighbouring cells to one another forming a second supracellular cytoskeletal network. Desmosomal cadherins (desmocollin, desmoglein) link the membranes of neighbouring cells and their cytoplasmic tails bind to the desmosomal plaque that anchors to IFs (reviewed in [50]). Desmosomal proteins turn over on a time-scale commensurate with IFs, far slower than adherens junction proteins [51]. Many genetic mutations affecting IFs or desmosomal proteins result in fragile epithelia [50], emphasizing their physiological importance. Despite this, their role in tissue mechanics remains understudied. Experiments on monolayers with ECM suggest that the IF network does not participate in setting tissue tension [52]. In suspended monolayers, keratin IFs appear to become tensed at high strain [10], perhaps underlying their non-linear stiffness. In vitro experiments on detached monolayers have shown that IFs dictate the ultimate strain at which intercellular contacts rupture [53].

At time-scales of tens of minutes to hours, cellular-scale phenomena such as re-arrangement, cell division, and cell extrusion become significant for tissue mechanics [54,55]. Collectively, these phenomena can dramatically remodel tissues as evidenced during many developmental processes such as convergent extension or germ band elongation. The impact of each of these cellular behaviours on tissue mechanics is strongly dependent upon their orientation with respect to the stress field (reviewed in $[11,30,55]$ ).

\section{Numerical models for understanding tissue mechanics}

To investigate the link between the tissue-scale mechanics and the molecular/cellular-scale organization and dynamics, researchers have increasingly been turning to computational 
models. Some models explicitly incorporate all potential actors of tissue rheology (reviewed in [56]) and these have given rise to platforms attempting to couple mechanics and signaling in great detail such as Chaste [57], Compucell3D [58] or Virtual cell [59]. However, this approach can become cumbersome because of the large number of parameters involved. A more economical design is to average the relevant biophysical processes occurring at smaller length-scales and faster time-scales than the phenomenon under consideration in the form of effective rheological parameters, an approach called homogenization in mechanics (Fig. 2). When the time- and length-scales of the participating phenomena are well separated, homogenization can represent a particularly efficient approach compared to more comprehensive models, both in terms of computational speed and understanding. For example, models seeking to understand the longer time-scales of tissue mechanics need not incorporate the dynamics of interstitial cytoplasmic flow or the actin cytoskeleton turnover because these will be in quasi-static equilibrium for time-scales longer than a few minutes.

A first class of models reduces cells to their centre of mass and considers pairwise interactions of particles subjected to forces such as short range repulsion due to contact inhibition [60] and long range attraction due to intercellular adhesion [61,62]. Such approaches have yielded insights into phenomena across various time-scales to understand collective cell dynamics [63], jamming where cells become caged by their neighbors [64], visco-plastic flows [65], and over longer time-scales, the emergence of an homeostatic state [66] viewed as a stable mechanical equilibrium between cell division and apoptosis. However, this approach cannot account for cell morphology and therefore fails to accurately describe the role of cell junctions in tissue mechanics.

To interpret changes in intercellular contact morphologies and sorting experiments [67], models incorporating intercellular adhesion energies, cortical tensions and a bulk elastic modulus emerged, such as vertex models (reviewed in [68]) and cellular Potts models [69]. Though these models often treat cells as two-dimensional objects, 3D formalisms exist [70] and can be extended to incorporate differences in active tensions between the apical and basal side [71] which are necessary to understand the spontaneous buckling observed in a number of tissues [71-73]. However, one current limitation of these models is the complexity of incorporating the wealth of rheological data observed experimentally.

In Engineering Sciences, materials are generally described at macroscopic scales as continua where the fine details of their underlying structures, such as the arrangement of individual atoms or defects in the microstructure, are lumped into effective material parameters. In the context of a cellularised aggregate, this involves coarse-graining the description at scales larger than the cell size. For instance, coarse graining of vertex models [74] leads to continuum models which incorporate active expansile and contractile regions corresponding to growth and contractility together with spontaneous curvature. These have been successfully used for modelling phenomena in which groups of cells are involved such as ventral furrow formation in flies [75], wound-healing [76], tissue invasion [77,78], growth of tumor spheroids [79], mechanical waves [80] and active 'turbulent' motion [81] in cellular sheets, somite formation [82], as well as spontaneous buckling and vilification in the intestine [83-85] or the brain [86]. The major drawback of these approaches has been the difficulty in deriving the coarse grained mechanical parameters directly from experiments and the challenge in linking them to biological phenomena. However, recent work comparing 
experimentally observed and predicted tissue dynamics has allowed to obtain relevant macroscopic rheological parameters [27].

\section{Conclusions}

In summary, cellularised materials present complex mechanical behaviours that depend on the time-scale and amplitude of the mechanical perturbation they are subjected to. Some aspects of the mechanical behaviours of cellularised materials may superficially appear similar to passive engineering materials but they in fact result from the complex dynamic interplay of multiple active energy-consuming processes. The challenge in the coming years will be to determine the relationship between molecular-scale organization/dynamics and tissue-scale mechanical properties.

\section{Acknowledgements}

NK was funded by the Rosetrees Trust, the UCL Graduate School, the EPSRC funded doctoral training program CoMPLEX. NK was in receipt of a UCL Overseas Research Scholarship. JF and PR are funded by BBSRC grant (BB/M003280 and BB/M002578) to GC and AK. GC is supported by a consolidator grant from the European Research Council (MolCellTissMech, agreement 647186). 


\section{References}

1. Davidson LA, Keller R, DeSimone DW: Assembly and remodeling of the fibrillar fibronectin extracellular matrix during gastrulation and neurulation in Xenopus laevis. Dev. Dyn. 2004, 231:888-895.

2. Davidson LA, Marsden M, Keller R, DeSimone DW: Integrin $\alpha 5 \beta 1$ and Fibronectin Regulate Polarized Cell Protrusions Required for Xenopus Convergence and Extension. Curr. Biol. 2006, 16:833-844.

3. George EL, Georges-Labouesse EN, Patel-King RS, Rayburn H, Hynes RO: Defects in mesoderm, neural tube and vascular development in mouse embryos lacking fibronectin. Development 1993, 119:1079-1091.

4. Leptin $M$, Bogaert $T$, Lehmann R, Wilcox $M$ : The function of PS integrins during Drosophila embryogenesis. Cell 1989, 56:401-408.

5. Philipps HM, Steinberg MS: Embryonic Tissues As Elastoviscous Liquids .1. Rapid and Slow Shape Changes in Centrifuged Cell Aggregates. J. Cell Sci. 1978, 30:1-20.

6. Forgacs G, Foty RA, Shafrir Y, Steinberg MS: Viscoelastic properties of living embryonic tissues: a quantitative study. . Biophys. J. 1998, 74:2227-34.

7. Marmottant P, Mgharbel A, Käfer J, Audren B, Rieu J-P, Vial J-C, van der Sanden B, Marée AFM, Graner $F$, Delanoë-Ayari $H$ : The role of fluctuations and stress on the effective viscosity of cell aggregates. Proc. Natl. Acad. Sci. U. S. A. 2009, 106:1727117275.

8. Guevorkian K, Colbert MJ, Durth M, Dufour S, Brochard-Wyart F: Aspiration of biological viscoelastic drops. Phys. Rev. Lett. 2010, 104:1-4.

9. Bonnet I, Marcq P, Bosveld F, Fetler L, Bellaiche Y, Graner F: Mechanical state, material properties and continuous description of an epithelial tissue. J. R. Soc. Interface 2012, 9:2614-2623.

10. Harris AR, Peter L, Bellis J, Baum B, Kabla AJ, Charras GT: Characterizing the mechanics of cultured cell monolayers . Proc. Natl. Acad. Sci. 2012, 109:1644916454.

11. Guillot C, Lecuit T: Mechanics of Epithelial Tissue. Science 2013, 340:1185-1189.

12. Brodland GW, Wiebe CJ: Mechanical effects of cell anisotropy on epithelia . Comput. Methods Biomech. Biomed. Engin. 2004, 7:91-99.

13. Gonzalez-Rodriguez D, Bonnemay L, Elgeti J, Dufour S, Cuvelier D, Brochard-Wyart F: Detachment and fracture of cellular aggregates . Soft Matter 2013, 9:2282.

14. Janmey PA, Schliwa M: Rheology. Curr. Biol. 2008, 18:639-641.

15. Tlili S, Gay C, Graner F, Marcq P, Molino F, Saramito P: Mechanical formalism for tissue dynamics . Eur. Phys. J. E 2015, 6:23.

16. Fabry B, Maksym GN, Butler JP, Glogauer M, Navajas D, Fredberg JJ: Scaling the microrheology of living cells. Phys. Rev. Lett. 2001, 87:1-4.

17. Oliver M, Kováts T, Mijailovich SM, Butler JP, Fredberg JJ, Lenormand G: Remodeling of integrated contractile tissues and its dependence on strain-rate amplitude. Phys. Rev. Lett. 2010, 105:1-4.

18. Fletcher DA, Mullins RD: Cell mechanics and the cytoskeleton . Nature 2010, 463:485-492.

19. Bausch AR, Kroy K: A bottom-up approach to cell mechanics . Nat. Phys. 2006, 2:231238.

20. Sato M, Levesque MJ, Nerem RM: Micropipette aspiration of cultured bovine aortic 
endothelial cells exposed to shear stress. Arteriosclerosis 2015, 7:276-286.

21. Hoffman BD, Crocker JC: Cell Mechanics: Dissecting the Physical Responses of Cells to Force. Annu. Rev. Biomed. Eng. 2009, 11:259-288.

22. Fritzsche M, Lewalle A, Duke T, Kruse K, Charras G: Analysis of turnover dynamics of the submembranous actin cortex. . Mol. Biol. Cell 2013, 24:757-67.

23. Mukhina S, Wang Y li, Murata-Hori M: $\alpha$-Actinin Is Required for Tightly Regulated Remodeling of the Actin Cortical Network during Cytokinesis. Dev. Cell 2007, 13:554-565.

24. Srivastava V, Iglesias PA, Robinson DN: Cytokinesis: Robust cell shape regulation . Semin. Cell Dev. Biol. 2015, doi:10.1016/j.semcdb.2015.10.023.

25. Hiraiwa T, Salbreux G: Role of Turnover in Active Stress Generation in a Filament Network. Phys. Rev. Lett. 2016, 116:188101.

26. Prost J, Jülicher F, Joanny J: Active gel physics . Nat. Phys. 2015, 11:131-139.

27. Machado PF, Duque J, Étienne J, Martinez-Arias A, Blanchard GB, Gorfinkiel N: Emergent material properties of developing epithelial tissues . BMC Biol. 2015, 13:98.

28. Salbreux G, Charras G, Paluch E: Actin cortex mechanics and cellular morphogenesis. Trends Cell Biol. 2012, 22:536-545.

29. Martin AC, Kaschube M, Wieschaus EF: Pulsed contractions of an actin-myosin network drive apical constriction. . Nature 2009, 457:495-9.

30. Heisenberg CP, Bellaïche $\mathrm{Y}$ : Forces in tissue morphogenesis and patterning. Cell 2013, 153.

31. Hoffman BD, Crocker JC: Cell mechanics: dissecting the physical responses of cells to force. Annu. Rev. Biomed. Eng. 2009, 11:259-288.

32. Ramms L, Fabris G, Windoffer R, Schwarz N, Springer R, Zhou C, Lazar J, Stiefel S, Hersch N, Schnakenberg $U$, et al.: Keratins as the main component for the mechanical integrity of keratinocytes. . Proc. Natl. Acad. Sci. U. S. A. 2013, 110:18513-8.

33. Seltmann K, Fritsch AW, Käs J a, Magin TM: Keratins significantly contribute to cell stiffness and impact invasive behavior. Proc. Natl. Acad. Sci. U. S. A. 2013, 110:18507-12.

34. Eckes B, Dogic D, Colucci-Guyon E, Wang N, Maniotis a, Ingber D, Merckling a, Langa $F$, Aumailley $M$, Delouvée a, et al.: Impaired mechanical stability, migration and contractile capacity in vimentin-deficient fibroblasts. J. Cell Sci. 1998, 111 ( Pt 1:1897-1907.

35. Wang N, Stamenović D: Contribution of intermediate filaments to cell stiffness, stiffening, and growth. Am. J. Physiol. Cell Physiol. 2000, 279:C188-C194.

36. Fudge DS, Winegard T, Ewoldt RH, Beriault D, Szewciw L, McKinley GH: From ultrasoft slime to hard ??-keratins: The many lives of intermediate filaments. Integr. Comp. Biol. 2009, 49:32-39.

37. Janmey PA, Euteneuer $U$, Traub P, Schliwa M: Viscoelastic properties of vimentin compared with other filamentous biopolymer networks. J. Cell Biol. 1991, 113:155160.

38. Kölsch A, Windoffer R, Würflinger T, Aach T, Leube RE, Kolsch A, Windoffer R, Wurflinger $T$, Aach $T$, Leube RE: The keratin-filament cycle of assembly and disassembly. . J. Cell Sci. 2010, 123:2266-2272.

39. Brangwynne CP, MacKintosh FC, Kumar S, Geisse NA, Talbot J, Mahadevan L, Parker 
KK, Ingber DE, Weitz DA: Microtubules can bear enhanced compressive loads in living cells because of lateral reinforcement. J. Cell Biol. 2006, 173:733-741.

40. Zhou J, Kim HY, Wang JH-C, Davidson L a: Macroscopic stiffening of embryonic tissues via microtubules, RhoGEF and the assembly of contractile bundles of actomyosin. Development 2010, 137:2785-2794.

41. Kollmannsberger P, Fabry B: Linear and Nonlinear Rheology of Living Cells. Annu. Rev. Mater. Res. 2011, 41:75-97.

42. Moeendarbary E, Valon L, Fritzsche M, Harris AR, Moulding D a, Thrasher AJ, Stride E, Mahadevan L, Charras GT: The cytoplasm of living cells behaves as a poroelastic material. . Nat. Mater. 2013, 12:253-61.

43. Delarue $M$, Joanny J-F, Julicher F, Prost J: Stress distributions and cell flows in a growing cell aggregate. Interface Focus 2014, doi:10.1098/rsfs.2014.0033.

44. Harris TJ, Tepass U: Adherens junctions: from molecules to morphogenesis . Nat Rev Mol Cell Biol 2010, 11:502-514.

45. Gumbiner BM: Regulation of cadherin activity. J. Cell Biol. 2000, 148:599-403.

46. Borghi N, Sorokina M, Shcherbakova OG, Weis WI, Pruitt BL, Nelson WJ, Dunn AR: Ecadherin is under constitutive actomyosin-generated tension that is increased at cell-cell contacts upon externally applied stretch . Proc. Natl. Acad. Sci. U. S. A. 2012, 109:12568-73.

47. Icard-Arcizet $D$, Cardoso O, Richert A, Hénon S: Cell stiffening in response to external stress is correlated to actin recruitment. Biophys. J. 2008, 94:2906-2913.

48. Guillot C, Lecuit T: Mechanics of Epithelial Tissue Homeostasis and Morphogenesis. Science (80-. ). 2013, 340:1185-1189.

49. Heisenberg C-P, Bellaïche Y: Forces in Tissue Morphogenesis and Patterning. Cell 2013, 153:948-962.

50. Johnson JL, Najor NA, Green KJ: Desmosomes: regulators of cellular signaling and adhesion in epidermal health and disease. Cold Spring Harb. Perspect. Med. 2014, 4:a015297.

51. Gloushankova NA, Wakatsuki T, Troyanovsky RB, Elson E, Troyanovsky SM: Continual assembly of desmosomes within stable intercellular contacts of epithelial A-431 cells. Cell Tissue Res. 2003, 314:399-410.

52. Harris AR, Daeden A, Charras GT: Formation of adherens junctions leads to the emergence of a tissue-level tension in epithelial monolayers. . J. Cell Sci. 2014, doi:10.1242/jcs.142349.

53. Huen AC, Park JK, Godsel LM, Chen X, Bannon LJ, Amargo E V., Hudson TY, Mongiu AK, Leigh IM, Kelsell DP, et al.: Intermediate filament-membrane attachments function synergistically with actin-dependent contacts to regulate intercellular adhesive strength. J. Cell Biol. 2002, 159:1005-1017.

54. Ranft J, Basan M, Elgeti J, Joanny J-F, Prost J, Jülicher F: Fluidization of tissues by cell division and apoptosis. Proc Natl Acad Sci U S A 2010, 107:20863-20868.

55. Wyatt T, Baum B, Charras G: A question of time: tissue adaptation to mechanical forces . Curr. Opin. Cell Biol. 2016, 38:68-73.

56. Brodland GW: How computational models can help unlock biological systems . Semin. Cell Dev. Biol. 2015, 47-48:62-73.

57. Mirams GR, Arthurs CJ, Bernabeu MO, Bordas R, Cooper J, Corrias A, Davit Y, Dunn SJ, Fletcher AG, Harvey DG, et al.: Chaste: An Open Source C++ Library for

Computational Physiology and Biology. PLoS Comput. Biol. 2013, 9. 
58. Swat MH, Thomas GL, Belmonte JM, Shirinifard A, Hmeljak D, Glazier JA: Multi-scale modeling of tissues using CompuCell3D. Methods Cell Biol. 2012, 110:325.

59. Resasco DC, Gao F, Morgan F, Novak IL, Schaff JC, Slepchenko BM: Virtual Cell: Computational tools for modeling in cell biology. Wiley Interdiscip. Rev. Syst. Biol. Med. 2012, 4:129-140.

60. Davis JR, Luchici A, Mosis F, Thackery J, Salazar JA, Mao Y, Dunn GA, Betz T, Miodownik M, Stramer BM: Inter-cellular forces orchestrate contact inhibition of locomotion. Cell 2015, 161:361-373.

61. Löber J, Ziebert F, Aranson IS: Collisions of deformable cells lead to collective migration. Sci. Rep. 2015, 5.

62. Deforet M, Hakim V, Yevick HG, Duclos G, Silberzan P: Emergence of collective modes and tri-dimensional structures from epithelial confinement. . Nat. Commun. 2014, 5:3747.

63. Palsson $\mathrm{E}$, Othmer HG: A model for individual and collective cell movement in Dictyostelium discoideum. . Proc. Natl. Acad. Sci. U. S. A. 2000, 97:10448-53.

64. Garcia S, Hannezo E, Elgeti J, Joanny J-F, Silberzan P, Gov NS: Physics of active jamming during collective cellular motion in a monolayer . Proc. Natl. Acad. Sci. U. S. A. 2015, 112:15314-15319.

65. Czirok A, Isai DG: Cell resolved, multiparticle model of plastic tissue deformations and morphogenesis. Phys. Biol. 2015, 12:16005.

66. Podewitz N, Delarue M, Elgeti J: Tissue homeostasis: A tensile state . EPL (Europhysics Lett. 2015, 109:58005.

67. Townes P, Holtfreter J: Directed movements and selective adhesion. J. Exp. Zool. 1955, 128:53-120.

68. Fletcher AG, Osborne JM, Maini PK, Gavaghan DJ: Implementing vertex dynamics models of cell populations in biology within a consistent computational framework. Prog. Biophys. Mol. Biol. 2013, 113:299-326.

69. Graner F, Glazier JA: Simulation of biological cell sorting using a two-dimensional extended Potts model. Phys. Rev. Lett. 1992, 69:2013.

70. Honda $\mathrm{H}$, Tanemura $\mathrm{M}$, Nagai $\mathrm{T}$ : A three-dimensional vertex dynamics cell model of space-filling polyhedra simulating cell behavior in a cell aggregate. J. Theor. Biol. 2004, 226:439-453.

71. Hannezo E, Prost J, Joanny J-F: Theory of epithelial sheet morphology in three dimensions. . Proc. Natl. Acad. Sci. 2014, 111:27-32.

72. Misra M, Audoly B, Kevrekidis IG, Shvartsman SY: Shape Transformations of Epithelial Shells . Biophys. J. 2016, 110:1670-1678.

73. Bielmeier C, Alt S, Weichselberger V, La Fortezza M, Harz H, Jülicher F, Salbreux G, Classen A-K: Interface Contractility between Differently Fated Cells Drives Cell Elimination and Cyst Formation . Curr. Biol. 2016, 2:563-574.

74. Murisic N, Hakim V, Kevrekidis IG, Shvartsman SY, Audoly B: From Discrete to Continuum Models of Three-Dimensional Deformations in Epithelial Sheets . Biophys. J. 2015, 109:154-163.

75. He B, Doubrovinski K, Polyakov O, Wieschaus E: Apical constriction drives tissuescale hydrodynamic flow to mediate cell elongation . Nature 2014, 508:392-396.

76. Cochet-Escartin O, Ranft J, Silberzan P, Marcq P: Border forces and friction control epithelial closure dynamics. Biophys J 2014, 106:65-73.

77. Ranft J, Aliee M, Prost J, Jülicher F, Joanny JF: Mechanically driven interface 
propagation in biological tissues. New J. Phys. 2014, 16.

78. Recho P, Ranft J, Marcq P: One-dimensional collective migration of a proliferating cell monolayer. Soft Matter 2016, 12:2381-2391.

79. Montel F, Delarue M, Elgeti J, Malaquin L, Basan M, Risler T, Cabane B, Vignjevic D, Prost J, Cappello G, et al.: Stress Clamp Experiments on Multicellular Tumor Spheroids . Phys. Rev. Lett. 2011, 107:188102.

80. Banerjee S, Utuje KJC, Marchetti MC: Propagating Stress Waves During Epithelial Expansion. Phys. Rev. Lett. 2015, 114:228101.

81. Doostmohammadi A, Adamer MF, Thampi SP, Yeomans JM: Stabilization of active matter by flow-vortex lattices and defect ordering . Nat. Commun. 2016, 7:10557.

82. Truskinovsky L, Vitale G, Smit TH: A mechanical perspective on vertebral segmentation . Int. J. Eng. Sci. 2014, 83:124-137.

83. Savin T, Kurpios NA, Shyer AE, Florescu P, Liang H, Mahadevan L, Tabin CJ: On the growth and form of the gut. Nature 2011, 476:57-62.

84. Shyer AE, Tallinen T, Nerurkar NL, Wei Z, Gil ES, Kaplan DL, Tabin CJ, Mahadevan L: Villification : How the Gut Gets Its Villi. Nature 2013, 342:6155.

85. Hannezo E, Prost J, Joanny JF: Instabilities of monolayered epithelia: Shape and structure of villi and crypts. Phys. Rev. Lett. 2011, 107:1-5.

86. Tallinen T, Chung JY, Rousseau F, Girard N, Lefèvre J, Mahadevan L: On the growth and form of cortical convolutions. Nat. Phys. 2016, [no volume]. 


\section{Papers of special interest:}

(Tlili et al Eur Phys J, 2015). The authors introduce a robust formalism for describing the rheology of living biological tissues at short and long time-scales.

(Prost et al, Nat Phys, 2015). The authors provide an up to date review of the formalism of active gel physics, its capabilities and applications.

(Machado et al, BMC Biol, 2015). This paper introduces a new theoretical and analytical framework to characterise the rheology of epithelial cells in the context of a tissue undergoing natural oscillations in cell shape during morphogenesis.

(Hannezo, PNAS, 2014). The authors describe a model based on cell surface tensions that provides a physical explanation for the various morphologies observed in epithelial sheets

(Bielmeier 2016). This paper reports on how morphogenesis can arise from differences in cell-fate and local clustering.

(He et al, Nature 2014). The authors show that the flows observed in the cytoplasm of Drosophila during ventral furrow formation can be understood from hydrodynamic theories and that cell individualisation in the epithelium is dispensable.

(Hiraiwa and Salbreux, 2016). The authors investigate how tension can be generated in isotropic actomyosin networks using computational approaches. 
Figures

A

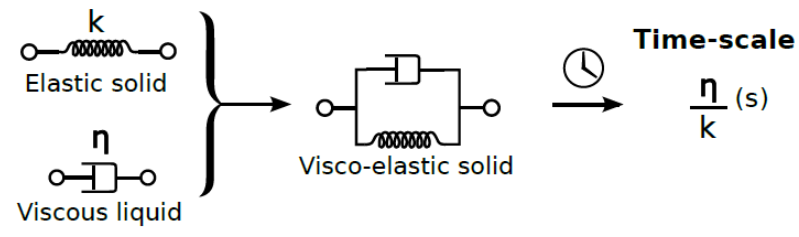

B

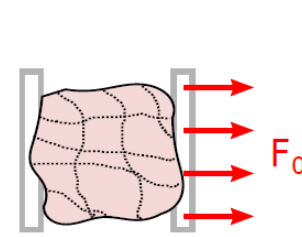

Material properties

of cellularized tissue

CREEP TEST

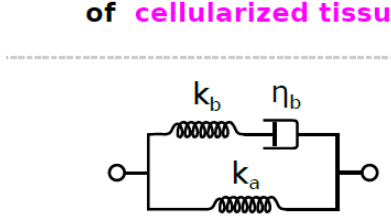

Standard linear solid

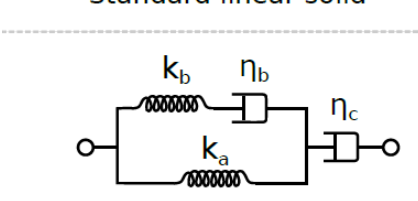

Modified Maxwell model (liquid)

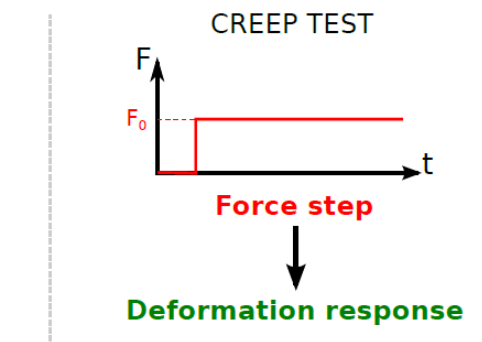

STRESS RELAXATION TEST

$\varepsilon$

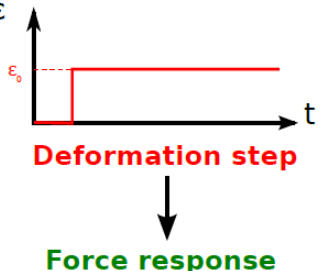

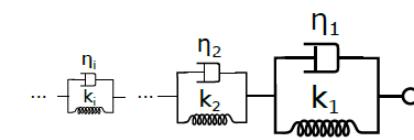

Power-law modified Kelvin model

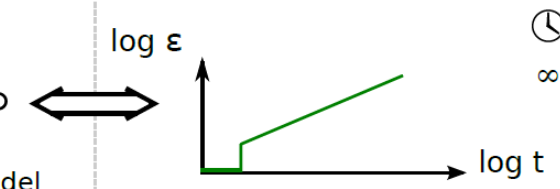
$\log t$ (a)

2

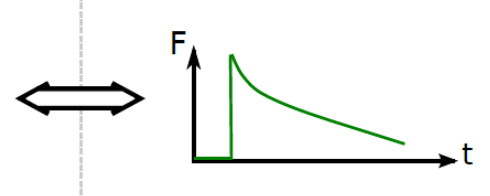

$\log t$

Figure 1:

\section{Rheological models of cellular aggregates.}

A) In rheological models, standard mechanical components include elastic springs and viscous dashpots. The stiffness $k$ of the spring sets the force necessary to extend it. When the force applied to a spring is removed, it returns to its initial shape thereby mimicking a solid. Dashpots are parametrized by a viscosity $\eta$ that sets the rate at which they can be deformed. In contrast to springs, they do not recover their initial length if the applied force becomes zero. Combinations of springs and dashpots can be used to model tissue responses. For example, a spring in parallel with a dashpot can mimic a viscoelastic solid whose characteristic time-scale is set by the ratio of the dashpot's viscosity $\eta$ to the spring elasticity k. B) Examples of common rheological models used to describe cellularised tissue dynamics. Two mechanical tests are commonly used to investigate the rheology of materials: creep tests and stress relaxation tests. The creep test measures the deformation of a material in response to a step force. Relaxation tests monitor the evolution of stress in response to a step deformation. Both tests are complementary in probing the system. For each model, the number of time-scales involved is indicated. The standard linear solid has been proposed to model cellularised materials which behave as solids at long time-scales. These are composed of a spring in series 
with a dashpot $\left(k_{b}, \eta_{b}\right)$, representing the rheology of cytoplasm arranged in parallel with another spring $\left(k_{a}\right)$, possibly emerging from myosin contractility. The addition of a dashpot $\left(\eta_{c}\right)$ in series with the standard linear solid can be used to mimic cell-cell rearrangements within a cellularised aggregate. In other experimental conditions, scale-free flow of the tissue has also been observed corresponding to the arrangement in series of large numbers of viscoelastic solids, each with a distinct stiffness and viscosity. 


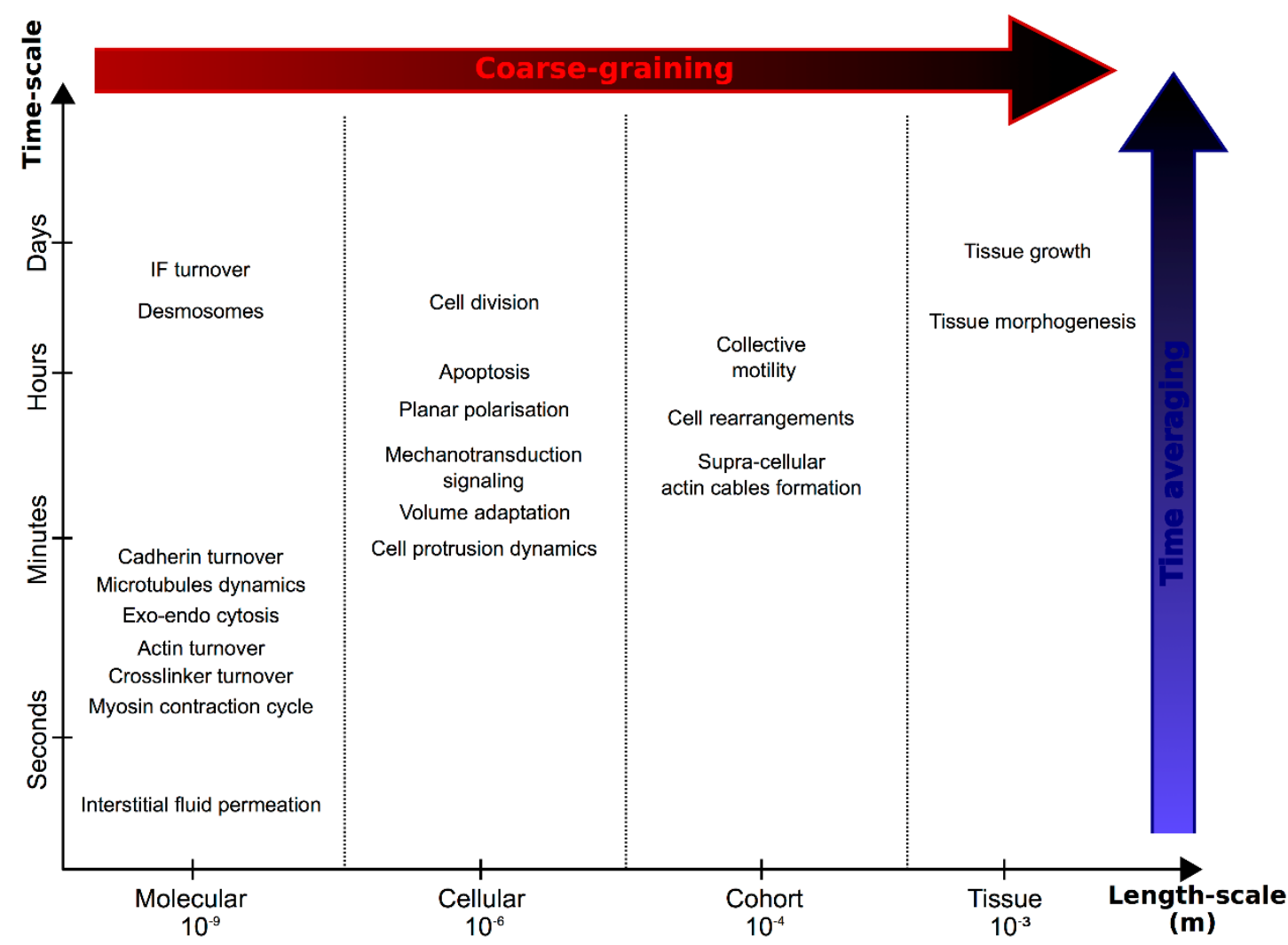

Figure 2:

Coarse graining and time averaging can guide which phenomena are included in modelling. Phenomena participating in cellularised aggregate rheology classified by length-scale and time-scale. Computational models can exclude structures smaller than a chosen scale, a process known as coarse graining (red arrow), and can seek to represent dynamics only above a chosen time-scale, a process known as time averaging (blue scale). Length-scales have been subdivided into the characteristic length-scales of molecules (Molecular), cells (Cellular), groups of cells (Cohort), and tissues. 